\title{
Nichtstun in der Medizin
}

Tun wir in der Medizin zu viel? Gelegentlich wohl schon. Oder tun wir in der Medizin zu wenig? Auch das kommt vor. Und sollten wir gegebenenfalls auch nichts tun? Ja, unbedingt - wir dürfen, wir sollten häufiger nichts tun.

\section{Beat Gerber}

Facharzt für Allgemeine Medizin, CAS Philosophie und Medizin

Bei diesem Text handelt es sich um eine stark gekürzte Fassung einer CAS-Qualifikationsarbeit für das Nachdiplomstudium in Philosophie und Medizin der Universität Luzern. Studienleiter und Dozent: Dr. Christian Neuhäuser.

\section{* Bei «Arzt» und «Patient» sind Frauen immer mitgemeint.}

Korrespondenz:

Dr. med. Beat Gerber

Schmiedmatte 5

CH-3665 Wattenwil

Tel. 0333563233

beat.gerber[at]hispeed.ch

\section{Nichtstun - eine Option}

Der Grundgedanke meiner Überlegungen über das «Nichtstun in der Medizin» entspringt meiner hausärztlichen Tätigkeit. Als ausschliesslich klassisch ausgebildeter und praktizierender Arzt fühle ich mich von meiner Herkunft her klar der Schulmedizin verbunden, und meine täglichen ärztlichen Beurteilungen und Behandlungen basieren auf ihr. Es ist wichtig, dass meine Gedanken zum Thema Nichtstun vor diesem Hintergrund gelesen und verstanden werden.

Der Grundgedanke dieser Arbeit ist die nicht seltene ärztliche Erfahrung, die zeigt, dass in gewissen Fällen Abklärungen und Behandlungen von Patienten nicht nur entbehrlich sind, sondern unser ärztliches Tun für deren Gesundheit und Heilung in der Retrospektive als suboptimal oder gar als kontraproduktiv beurteilt werden muss - oder kurz gesagt: Das Tun, unser Tun, war letztlich nicht im Sinn des Patienten, es war falsch.

Nun ist aber der Aspekt des Unterlassens in unserer beruflichen Tätigkeit und im Verständnis unserer westlichen Gesellschaft ungewohnt und per se suspekt - und solches Tun, oder eben Nicht-tun, ist schwierig zu rechtfertigen und noch schwieriger $\mathrm{zu}$ verantworten.

Rein intuitiv spürt jeder praktizierende Arzt*, dass situativ das "Nichtstun", das bewusst passive Verhalten, das dem spontanen Verlauf des Krankheitsgeschehens den Vortritt gibt, in der ärztlichen Betreuung von Kranken eine verantwortungsvolle Haltung und sogar ein zentraler Auftrag ärztlicher Tätigkeit sein kann. Dieses «Nichtstun» befindet sich im heutigen medizinischen Diskurs allerdings in marginaler Position, da ein solches aus verschiedenen Gründen für Arzt und Patient kaum zur Anwendung kommt: teils aus Angst vor dem Vorwurf, einer modernen, interventionellen Medizin nicht zu genügen, teils aber auch bedingt durch die Gefahr und das Risiko, bei Nichthandeln der ärztlichen Hilfeleistungspflicht nicht gerecht zu werden. Beides sind Wagnisse, denen sich ein ambitionierter und dem Fortschrittsverständnis verpflichteter Arzt nicht aussetzen will. Dieser lässt sich unter diesem Handlungsdruck gerne zu einem gewissen Aktivismus, des Öfteren auch Hyperaktivismus, verleiten, immer im ehrlichen und selbstlosen Interesse, für seinen Patienten in jeder Situation das Denkbare gedacht und das Machbare gemacht zu haben.
Und so habe ich die folgende These aufgestellt: «Nichtstun» im Sinne der Unterlassung jeglicher weiterer diagnostischer und therapeutischer Interventionen im Krankheitsfall ist situativ ehrlich und konsequent und kann (und muss) dann im Interesse des Patienten sein, wenn Interventionen jeglicher Art für das körperliche oder seelische Wohl des Patienten kontraproduktiv sind. Argumentativ lässt sich diese These folgendermassen begründen:

1. Wenn diagnostische und therapeutische Absenz im Sinne des Patienten ist, wenn dem Spontanverlauf gegenüber jeglicher medizinischer Einflussnahme der Vortritt gelassen wird, weil der Spontanverlauf die für den Patienten bessere Alternative ist, dann sind wir dem Patienten, der Gesellschaft, aber auch uns gegenüber verpflichtet, das medizinische Tun zu unterlassen. Zusätzlich und darüber hinaus gibt es aber das menschliche Tun; gemeint sind das anteilnehmende, stützende Präsentsein und das sympathisch-mitfühlende Begleiten. Beides ist erwartungsgemäss immer geboten und steht hier nicht zur Diskussion. Selbstverständlich kann es auch nicht um die Unterlassung notwendiger Schmerztherapien oder gar palliativer Therapien gehen.

2. Ökonomische Gründe für ein «Nichtstun» in der Medizin könnten zwar, sollen aber bei meiner Argumentation explizit nicht beigezogen werden. Es geht hier zwingend nicht um den ökonomischen Aspekt, da meiner Meinung nach situatives Unterlassen von diagnostischem und therapeutischem Tun ganz und gar zum wahren ärztlichen Handeln gehören muss - oder mit Martin Heidegger gesprochen: Situativ praktiziertes Unterlassen soll Ausdruck einer «eigentlichen Existenzweise» der ärztlichen Tätigkeit sein und sich damit klar von einer «uneigentlichen, alltäglichen Existenzweise», nämlich dem sinnlosen Tun, abgrenzen.

3. Situatives Unterlassen von medizinischem Tun, insbesondere das Unterlassen von medizintechnischem «Dazutun», schafft unersetzbare Freiräume für die in manchen Situationen dringend notwendige Krankheitsverarbeitung und eine gegebenenfalls erwünschte Sterbevorbereitung. Von jeder nicht wirklich indizierten medizinischen Vereinnahmung ist in jeder beliebigen Gesundheits- und Krankheitssituation abzusehen, 
würde eine solche, laut der Ethikerin Monika Bobbert, doch einem der vier moralischen Autonomierechte eines jeden Patienten widersprechen [1]

\section{Das westliche Nichtstun - «Negative Ethik»}

«Negative Ethik» ist ein Begriff, der 1988 von Henning Ottmann kreiert wurde, damals unter dem Eindruck der Grossrisiken der industriellen Gesellschaft und der alltäglichen Übel des sogenannten Fortschritts. Ottmanns Negative Ethik besagt, dass bereits die traditionelle Ethik, neben einer Ethik des Handelns, immer auch eine Ethik des Nicht-Handelns ist, und dass das sekundäre Interesse am Nicht-Handeln heute nicht mehr reicht. Er fordert eine Negative Ethik, für die das Nicht-Tun und das Lassen das Primäre, das Handeln aber das Sekundäre ist [2]. Negative Ethik geht von der Prämisse aus, dass sich das Tun erklären muss, und nicht das Nicht-Tun. Negative Ethik fragt: Was sollen wir besser lassen? Dabei darf Negative Ethik nie mit der Aufforderung zur Faulheit oder Nachlässigkeit verstanden werden. Die skeptische Ethik von Wilhelm Weischedel nennt in diesem Sinne als «ethische Grundhaltungen auf dem Grunde der Abschiedlichkeit» folgende Tugenden: Entsagung, Selbstbescheidung, Besonnenheit und, am wichtigsten, die Gelassenheit, die zur Kardinaltugend der modernen Welt aufsteigt [3]. Und auch Aristoteles, so Ottmann, habe schon gewusst, dass nicht die Arbeit, sondern erst die Musse dem Leben Sinn verschafft [4].

\section{«Negative Ethik fragt: Was sollen wir besser lassen?»}

Die Philosophie der Negativen Ethik spielt uns den Gedanken zu, dass es auch eine Ethik des Nichthandelns gibt und diese konsequent auf den Bereich der Medizin übertragen werden kann.

\section{Das östliche Nichtstun - «Wu wei»}

Eine elementare Grundlage, die sich aus dem Wesen des Dao ergibt, ist das «Wu wei», das Nicht-Handeln, das Nicht-Eingreifen, basierend auf der Einsicht, dass das Dao selbst zum Ausgleich aller Kräfte drängt.

Was versteht man unter Dao? Es ist dem Daoismus nach gar nicht recht zu beschreiben, und wenn man es trotzdem versucht, dann könnte annähernd Folgendes darunter verstanden werden: Es ist aller Dinge Ursprung und Ziel, der Grund der Dinge. In gewissem Sinne also ein universal ontologischer Begriff der östlichen Philosophie.

Dadurch, dass das Dao selbst zum Ausgleich aller Kräfte drängt, wird die optimale Lösung von selbst und ohne menschliche Beihilfe entstehen. Für Laotse, dem mythischen Begründer des philosophischen

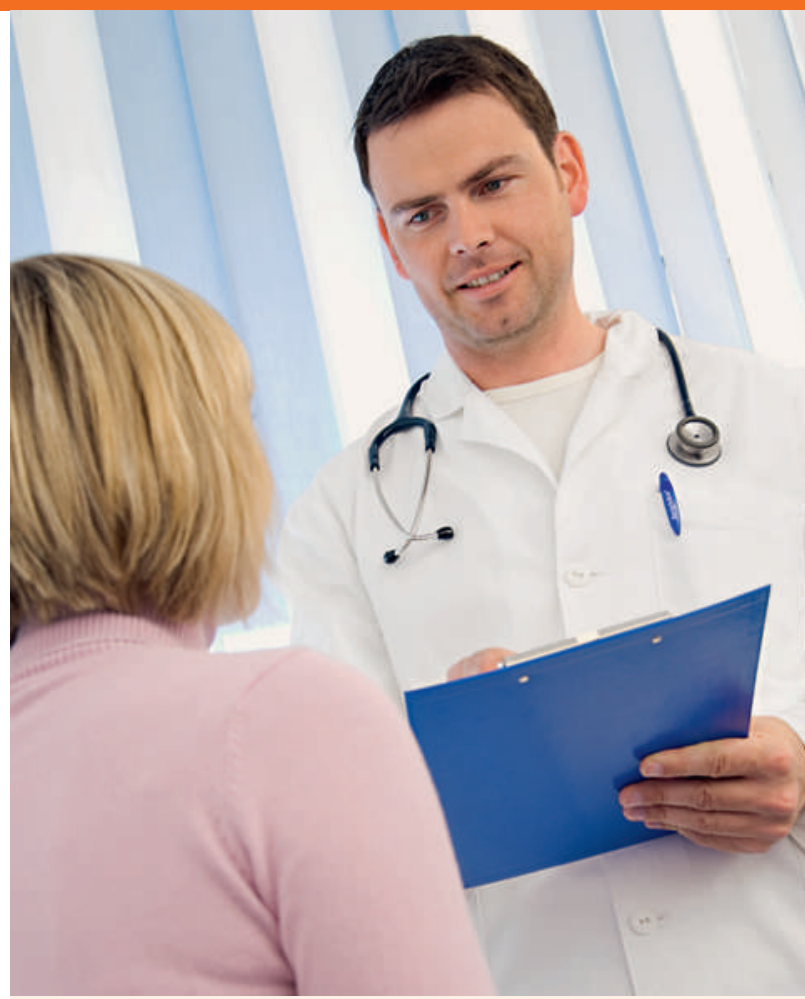

Eine schwere Vorstellung sowohl für Ärzte als auch für Patienten: abwarten und erst einmal nichts tun.

Daoismus, ist somit alles Tun ein bewusstes, vom Menschen initiiertes und aktives Abweichen vom natürlichen Gleichgewicht, eine menschliche Masslosigkeit [5].Jede Abweichung hat eine passive Gegenbewegung zur Folge, die das gestörte Gleichgewicht wiederherzustellen sucht.

Mit Wu wei gemeint ist das Prinzip des geringsten Widerstandes, die konsequente Nichteinmischung in den natürlichen Verlauf. Die Umstände bestimmen das Verhalten, der Daoist horcht auf die innere Stimme. Kraft wird nie Gegenkraft entgegengesetzt, vielmehr wird sie durch Nachgeben buchstäblich entkräftet. Menschliche Korrekturen und Verbesserungsversuche sind unsinnige Bemühungen und führen zu Chaos, nur Nichthandeln führt zum Erfolg [6].

Das chinesische «Wu wei» ist die wohl früheste und ursprünglichste philosophische Form des «Nichtstun», - im Sinne des situativen Nicht-Eingreifens in jegliche Spielart von spontanem Verlauf.

\section{Situatives Nichtstun in der Medizin als ärztliches Handeln}

Situatives Nichtstun in der Medizin steht in Verbindung mit Ehrlichkeit, Redlichkeit und Konsequenz, mit essentiellen Tugenden also, die im Kontext eines hilfesuchenden, kranken Menschen geboten sind. Auch Genügsamkeit und Einsicht sind hier zwingend, denn der Verzicht auf eine Intervention setzt das Akzeptieren eines Status quo voraus, - ebenso auch die Kraft und Stärke, die Fakten zu akzeptieren. Auch wenn dies bedeutet, das Ende eines Traumes, der Leben heisst, hinzunehmen, - einem Aufwachen gleich, zum letzten Schlaf: der Tod als Ort und Zu- 
stand, der weder Ort noch Zustand ist, wodurch das Nichtstun zum Paradigma des Nichts wird. Nichtstun, ein wahrlich ganz besonderes Tun.

Was für viele andere Berufe gilt, trifft ebenfalls für den Arztberuf zu: Ärztliches Handeln ist auch heute noch stark geprägt vom Lernen am Beispiel. Dieser Lernprozess bezieht sich nicht nur auf Wissen und Vermittlung von medizinischen Handlungen an sich, sondern auch auf allgemeine ärztliche Haltungen wie Fürsorge, Wahrhaftigkeit und Gerechtigkeit. Allge-
Dass aktuell jedoch das Tun gegenüber dem Nichtstun in seiner täglichen Umsetzung einen markant einfacheren Stand hat, dies zeigt der medizinische Alltag. Und dass hier Handlungsbedarf besteht, sollte in diesem Beitrag aufgezeigt worden sein.

Die in der heutigen Zeit und in unserer Denkweise oft übersehene und zurückgedrängte Strategie der Nicht-Intervention muss im Interesse einer ganzheitlichen und patientenorientierten Medizin eine hohe, bedingungslose und vor allem zweifelsfreie

\section{«Gefordert wird eine ausgewogene und paritätische Praxis des Handelns} und des Nicht-Handelns.»

meine Prinzipien wie Respekt vor dem Willen des Patienten und Nicht-Schaden müssen explizit ergänzt werden durch die situativ indizierte Option des «Nichtstun», und dies nicht nur im Sinne einer knapp geduldeten Akzeptanz, sondern als etablierte Möglichkeit einer ethisch korrekten und adäquaten Haltung des zuständigen Arztes.

\section{Was heisst das für die Praxis?}

Im Alltag der Medizin werden Patient und Arzt immer wieder mit der Frage konfrontiert, ob diagnostisch oder therapeutisch interveniert werden soll und muss, oder ob «Nichtstun» im Sinne des Patienten ist, da Interventionen jeglicher Art für das körperliche und seelische Wohl hinderlich sein könnten. In der Praxis erst wird sich zeigen, dass weder das permanente ärztliche Tun noch das Unterlassen ihre uneingeschränkte Richtigkeit haben, und dass ihre situative Gültigkeit, ihre erforderliche Gewichtung und ihre adäquate Umsetzbarkeit nur in einem Gespräch zwischen dem Patienten und dem Arzt gefunden werden kann. Dass hier nie auf irgendein erforderliches oder gebotenes Tun verzichtet werden darf, ist mindestens so fraglos, wie dass kein sinnloses Tun um des Tuns Willen praktiziert werden soll.

Gefordert wird eine ausgewogene und paritätische Praxis des Handelns und des Nicht-Handelns, die immer das Optimale für den Patienten im Visier hat. Weder dem Tun noch dem Unterlassen steht ein Primat zu, denn beide Thesen basieren auf einer verengten Perspektive, die in ihrer Exklusivität der Problematik und ihrer Lösung nicht gerecht wird.
Praktikabilität erlangen. Sowohl die Negative Ethik aus dem westlichen Gedankengut als auch das alte chinesische $\mathrm{Wu}$ wei aus dem östlichen denken und leben vor, was damit gemeint ist und wie in der Praxis damit umgegangen werden kann.

Das ärztliche Selbstverständnis erfordert, neu über das Arzt-Patienten-Verhältnis zu reflektieren. Wir sind gezwungen, über ethische Normen ärztlicher Tätigkeit ausreichend Kenntnis zu haben und diese gegebenenfalls zum Wohle des Patienten neu zu interpretieren, sowie moralische Beurteilungen zu kommunizieren und diese auch zu begründen. Wenn aus ethischen Gründen das «Nichtstun» gefragt ist, sind wir verpflichtet, nichts zu tun. Und zwar immer dann, wenn Begleiten besser als Intervenieren ist. Voraussetzung dafür ist, dass die Ärzteschaft und die medizinischen Wissenschaften, aber auch die Gesellschaft taugliche Rahmenbedingungen schaffen.

\section{Literatur}

1 Monika Bobbert M. Patientenautonomie und Pflege, Frankfurt; 2002.

2 Ottmann H. Negative Ethik. Berlin; 2005.

3 Weischedel W. Skeptische Ethik. Frankfurt; 1976. S. 209ff., zitiert nach Ottmann 2005 (s. [2], S. 8).

4 Ottmann [2] S. 10.

5 Laotse S. Tao Te King. Das Buch vom Sinn und Leben. Übersetzt und mit einem Kommentar von Richard Wilhelm. Kreuzlingen/München; 2004.

6 Dazu: Fischer T. Wu wei, Die Lebenskunst des Tao. Reinbek bei Hamburg; 1997. 